\title{
Continuous process improvement in the manufacture of carfilzomib. Part 2: An improved process for synthesis of the epoxyketone warhead
}

Matthew G. Beaver, ${ }^{*}$ Xianqing Shi, Jan Riedel, Parth Patel, Alicia Zeng, Michael T. Corbett, Jo Anna Robinson, Andrew T. Parsons, Sheng Cui, Kyle Baucom, Mike Lovette, Elçin Içten, Derek B. Brown, Ayman Allian, Tawnya G. Flick, Wendy Chen, Ning Yang, Shawn D. Walker

Process Development, Amgen, Inc.

360 Binney Street, Cambridge, Massachusetts 02142 (USA)

\section{Supporting Information}

\section{Table of Contents}

I. Synthesis of catalyst complex C2

II. Identification of conditions for Mn-catalyzed epoxidation
$S-1$

$S-2$

\section{Synthesis of catalyst complex C2}

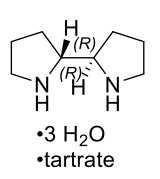

s1
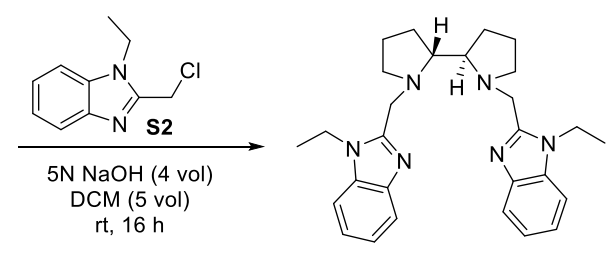

S3

Ligand S3. To a biphasic solution of $(2 R)-2-[(2 R)$-pyrrolidin-2-yl]pyrrolidine $\bullet$ trihydrate $\bullet$ tartrate S1 (10.0 g, $29.0 \mathrm{mmol}$ ), dichloromethane $(50 \mathrm{ml}, 5 \mathrm{ml} / \mathrm{g}$ ), and $5 \mathrm{~N}$ sodium hydroxide $(40 \mathrm{ml}, 4 \mathrm{ml} / \mathrm{g}$ ) was added 2(chloromethyl)-1-ethyl-benzimidazole $\mathbf{S 2}(11.4 \mathrm{~g}, 58.3 \mathrm{mmol})$ and the reaction mixture allowed to stir for $>16 \mathrm{~h}$ at ambient temperature. The layers were separated, the organic layer dried over $\mathrm{Na}_{2} \mathrm{SO}_{4}$, and then concentrated in vacuo to afford ligand $\mathbf{S 3}$ as a colorless oil $(13.1 \mathrm{~g}, 99 \%$ yield) with spectra identical to that previously reported in the literature. ${ }^{1}$

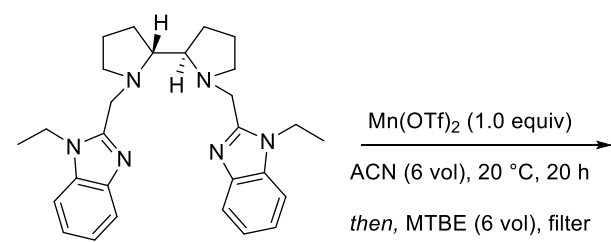

S3

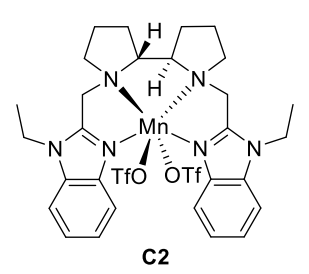

C2 
Complex C2. To solution of 1-ethyl-2-[[(2R)-2-[(2R)-1-[(1-ethylbenzimidazol-2-yl)methyl]pyrrolidin-2yl]pyrrolidin-1-yl]methyl]benzimidazole $\mathbf{S 3}(13.1 \mathrm{~g}, 28.8 \mathrm{mmol})$ in acetonitrile $(13 \mathrm{ml}, 1.0 \mathrm{ml} / \mathrm{g})$ was added a solution of manganese bis(trifluoromethanesulfonate) $(10.2 \mathrm{~g}, 28.8 \mathrm{mmol})$ in acetonitrile (66 $\mathrm{ml}, 5.0 \mathrm{ml} / \mathrm{g}$ ) and the reaction mixture was allowed to stir for $1 \mathrm{~h}$ at ambient temperature. To the resulting slurry was added methyl tert-butyl ether $(79 \mathrm{ml}, 6.0 \mathrm{ml} / \mathrm{g})$ over $2 \mathrm{~h}$. The slurry was filtered and the wet cake washed with 1:1 (v/v) ACN/MTBE $(40 \mathrm{ml}, 3.0 \mathrm{ml} / \mathrm{g})$, twice with MTBE $(40 \mathrm{ml}, 3.0 \mathrm{ml} / \mathrm{g})$, and dried under vacuum to afford complex C2 $(18.4 \mathrm{~g}$, 78\% yield) as a white crystalline solid with high resolution mass spectra matching that previously reported in the literature. ${ }^{1}$

\section{Identification of Conditions for Mn-Catalyzed Epoxidation}

The following tables and summary text provide a representative overview of the reaction conditions investigated toward optimization of the Mn-catalyzed epoxidation reaction of enone 2. Control experiments demonstrated that all reagents of the standard reaction conditions were required to achieve acceptable levels of conversion and diastereoselectivity.
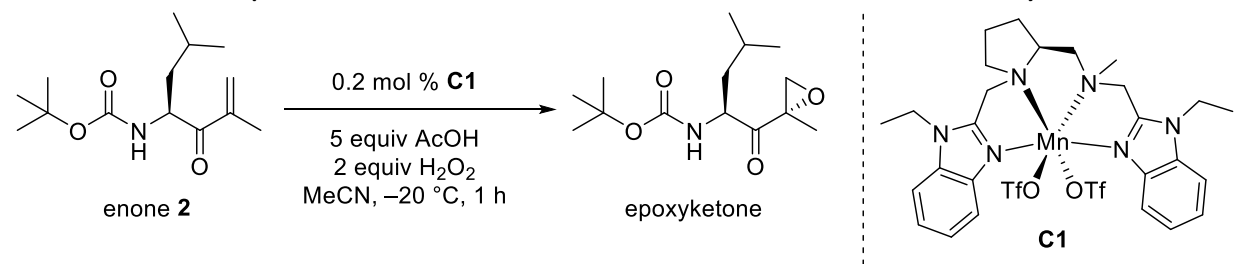

Table S1. Control experiments

\begin{tabular}{|c|l|c|c|}
\hline entry & condition & Assay yield $^{a}$ & d.r. $^{a}$ \\
\hline 1 & standard conditions & $78 \%$ & $88: 12$ \\
\hline 2 & no metal & $<1 \%$ & --- \\
\hline 3 & no ligand & $<1 \%$ & --- \\
\hline 4 & no oxidant & $<1 \%$ & --- \\
\hline 5 & no water & & --- \\
\hline 6 & no acetic acid & $17 \%$ & --- \\
\hline
\end{tabular}

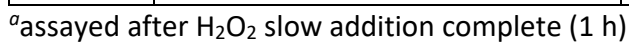

${ }^{b} \mathrm{H}_{2} \mathrm{O}_{2}$ urea adduct used as oxidant 


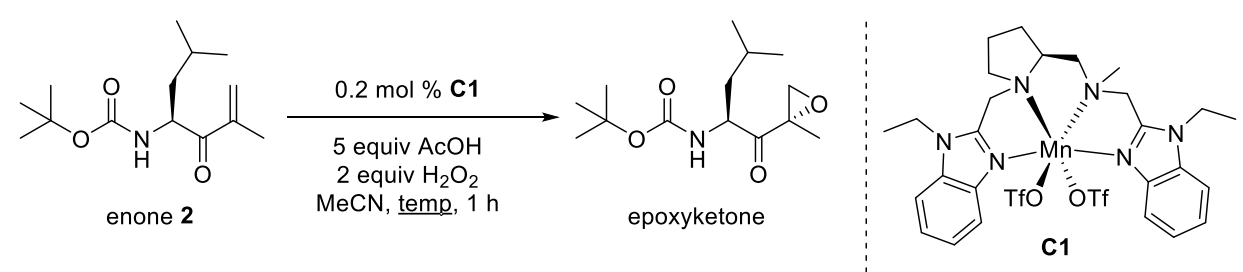

Table S2. Effect of reaction temperature

\begin{tabular}{|c|l|c|c|c|}
\hline entry & temperature & assay yield $^{a}$ & conversion $^{a}$ & d.r. $^{a}$ \\
\hline 1 & $10{ }^{\circ} \mathrm{C}$ & $83 \%$ & $87 \%$ & $84: 16$ \\
\hline 2 & $0{ }^{\circ} \mathrm{C}$ & $86 \%$ & $93 \%$ & $85: 15$ \\
\hline 3 & $-10{ }^{\circ} \mathrm{C}$ & $91 \%$ & $100 \%$ & $87: 13$ \\
\hline 4 & $-20{ }^{\circ} \mathrm{C}$ & $78 \%$ & $91 \%$ & $88: 12$ \\
\hline 5 & $-40{ }^{\circ} \mathrm{C}$ & $79 \%$ & $80 \%$ & $90: 10$ \\
\hline
\end{tabular}

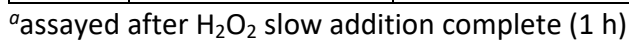<smiles>C=C(C)C(=O)C(CC(C)C)NC(=O)OC(C)(C)C</smiles>

enone 2

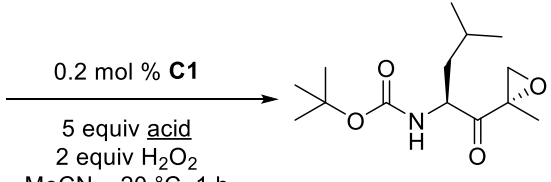

epoxyketone

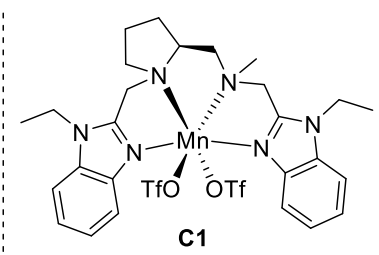

Table S3. Effect of acid additive

\begin{tabular}{|c|l|c|c|c|}
\hline entry & acid & assay yield $^{a}$ & conversion $^{a}$ & d.r. $^{a}$ \\
\hline 1 & acetic acid & $78 \%$ & $91 \%$ & $88: 12$ \\
\hline 2 & 2-ethyl-hexanoic acid & $6 \%$ & n.d. & $86: 14$ \\
\hline 3 & Pivalic acid & $79 \%$ & $94 \%$ & $87: 13$ \\
\hline 4 & 4-trifluoromethylbenzoic acid* & $>1 \%$ & $>1 \%$ & -- \\
\hline 5 & benzoic acid & \\
\hline 6 & trifluoro acetic acid & $1 \%$ & $40 \%$ & $86: 14$ \\
\hline 7 & propionic acid & $8 \%$ & $83 \%$ & $88: 12$ \\
\hline 8 & 1-adamantine carboxylic acid* & $53 \%$ & n.d. & n.d. \\
\hline
\end{tabular}

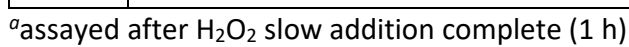

${ }^{b}$ poor solubility in $\mathrm{MeCN}$ 

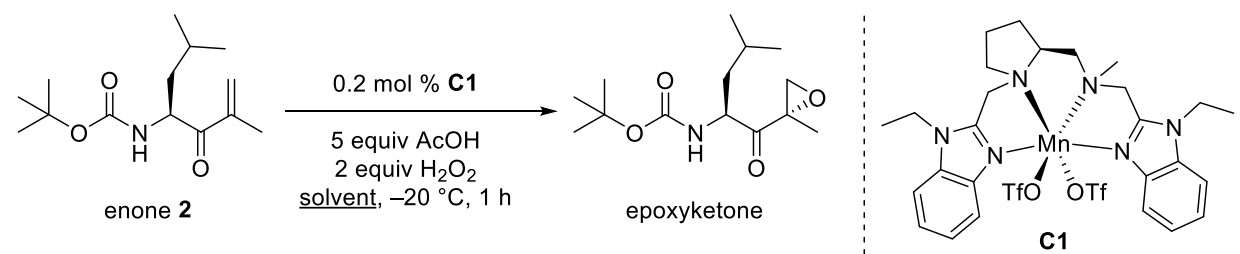

Table S4. Effect of reaction solvent

\begin{tabular}{|l|l|c|c|c|}
\hline entry & acid & assay yield & conversion $^{a}$ & d.r. $^{a}$ \\
\hline 1 & acetonitrile & $78 \%$ & $91 \%$ & $88: 12$ \\
\hline 2 & propionitrile & $62 \%$ & $78 \%$ & $87: 13$ \\
\hline 3 & $n$-butyronitrile & $19 \%$ & $30 \%$ & $88: 12$ \\
\hline 4 & methoxyaceto-nitrile & $59 \%$ & $73 \%$ & $88: 12$ \\
\hline 5 & cyclopropane-carbonitrile & $37 \%$ & $47 \%$ & $87: 13$ \\
\hline
\end{tabular}

assayed after $\mathrm{H}_{2} \mathrm{O}_{2}$ slow addition complete (1 h)
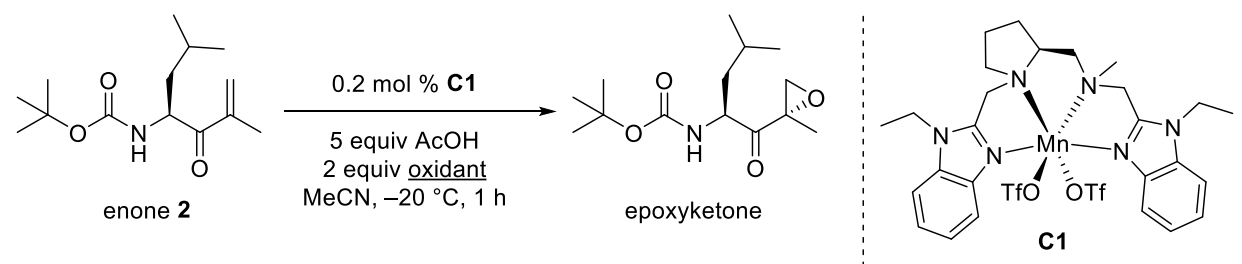

Table S5. Effect of oxidant identity

\begin{tabular}{|l|l|c|c|c|}
\hline entry & oxidant & assay yield $^{a}$ & conversion $^{a}$ & d.r. $^{a}$ \\
\hline 1 & $50 \% \mathrm{H}_{2} \mathrm{O}_{2}$ & $78 \%$ & $98 \%$ & $88: 12$ \\
\hline 2 & $m \mathrm{CPBA}$ & $2 \%$ & $40 \%$ & $57: 43$ \\
\hline 3 & $\mathrm{AcOOH}$ & $9 \%$ & $47 \%$ & $87: 13$ \\
\hline 4 & $\mathrm{PhIOAC}_{2}$ & $>1 \%$ & $77 \%$ & --- \\
\hline 5 & $t \mathrm{BuOOH}$ in water & $37 \%$ & $47 \%$ & $87: 13$ \\
\hline 6 & $t \mathrm{BuOOH}$ in decane & $40 \%$ & $55 \%$ & $87: 13$ \\
\hline 7 & $\mathrm{NaOCl}$ & $>1 \%$ & $10 \%$ & --- \\
\hline 8 & $\mathrm{PhIO}^{2}$ & $16 \%$ & $27 \%$ & $88: 12$ \\
\hline 9 & $30 \% \mathrm{H}_{2} \mathrm{O}_{2}{ }^{b}$ & $86 \%$ & $100 \%$ & $90: 10$ \\
\hline 10 & $50 \% \mathrm{H}_{2} \mathrm{O}_{2}{ }^{b}$ & $81 \%$ & $100 \%$ & $90: 10$ \\
\hline
\end{tabular}

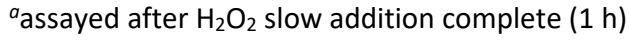

${ }^{b}$ with ligand $\mathbf{C 2}$ 


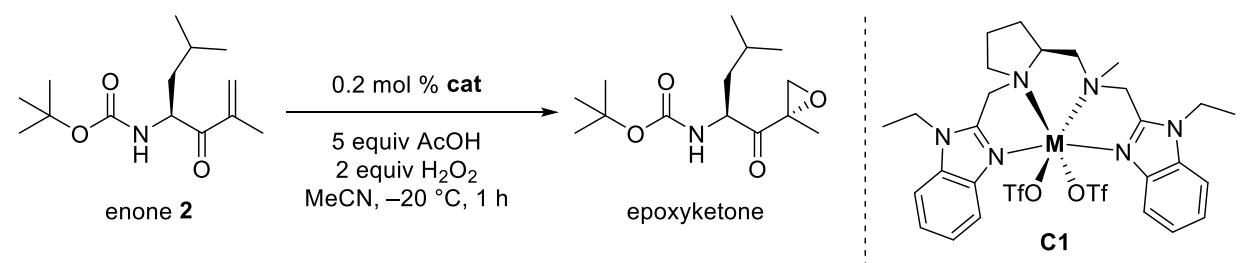

Table S6. Effect of metal catalyst

\begin{tabular}{|l|l|c|c|c|}
\hline entry & metal & assay yield $^{a}$ & conversion $^{a}$ & d.r. $^{a}$ \\
\hline 1 & $\mathrm{Fe}(\mathrm{OTf})_{2}$ & $59 \%$ & $100 \%$ & $86: 14$ \\
\hline 2 & $\mathrm{Cu}(\mathrm{OAc})_{2}$ & $>1 \%$ & $23 \%$ & --- \\
\hline 3 & $\mathrm{Co}(\mathrm{OAc})_{2}$ & $>1 \%$ & $22 \%$ & --- \\
\hline 4 & $\mathrm{Eu}(\mathrm{OTf})_{3}$ & $>1 \%$ & $25 \%$ & --- \\
\hline 5 & $\mathrm{Mn}(\mathrm{OAc})_{2}{ }^{b}$ & $45 \%$ & $68 \%$ & $88: 12$ \\
\hline 6 & $\mathrm{FeCl}$ & $>1 \%$ & $25 \%$ & --- \\
\hline 7 & $\mathrm{Fe}(\mathrm{OTf})_{3}$ & $58 \%$ & $100 \%$ & $87: 13$ \\
\hline
\end{tabular}

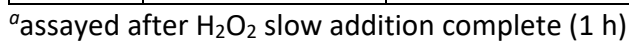

${ }^{b}$ poor solubility in $\mathrm{MeCN}$
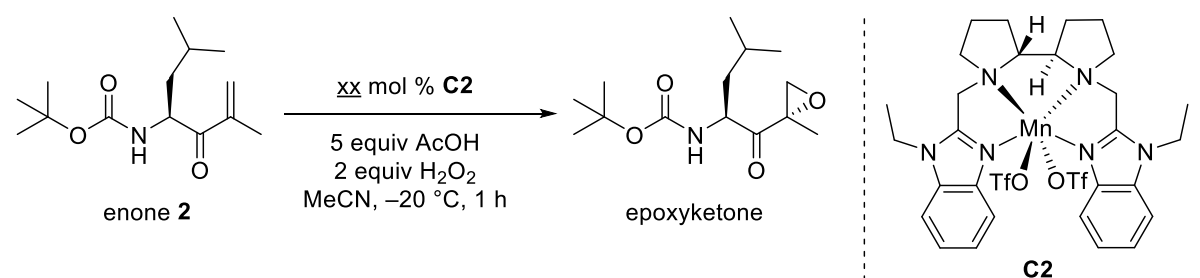

Table S7. Effect of catalyst loading

\begin{tabular}{|l|l|c|c|c|}
\hline entry & cat. loading & assay yield $^{a}$ & conversion $^{a}$ & d.r. $^{a}$ \\
\hline 1 & $0.2 \mathrm{~mol}^{6}$ & $81 \%$ & $100 \%$ & $90: 10$ \\
\hline 2 & $0.1 \mathrm{~mol} \%$ & $85 \%$ & $100 \%$ & $90: 10$ \\
\hline 3 & $0.02 \mathrm{~mol} \%$ & $80 \%$ & $88 \%$ & $91: 9$ \\
\hline 4 & $0.01 \mathrm{~mol} \%$ & $35 \%$ & $43 \%$ & $90: 10$ \\
\hline 5 & $0.002 \mathrm{~mol} \%$ & $3 \%$ & $8 \%$ & $89: 11$ \\
\hline
\end{tabular}

assayed after $\mathrm{H}_{2} \mathrm{O}_{2}$ slow addition complete (1 h)

${ }^{b}$ product decomposition observed 
A brief summary of ligand architecture is described below. All screening was conducted with both $(S)$ - and $(R)$-enone to evaluate matched/mismatched pairings; only the best result is shown. A solution of ligand and $\mathrm{Mn}(\mathrm{OTf})_{2}$ in $\mathrm{ACN}$ was prepared prior to use.<smiles>C=C(C)C(=O)C(CC(C)C)NC(=O)OC(C)(C)C</smiles><smiles>CCn1c(CN(C)C2CCCC2c2nc3ccccc3n2CC)nc2ccccc21</smiles>

c1

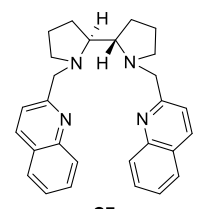

C7

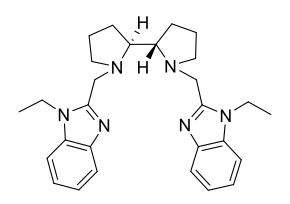

c2

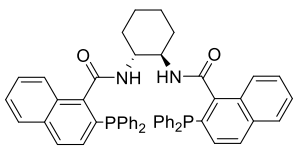

C8

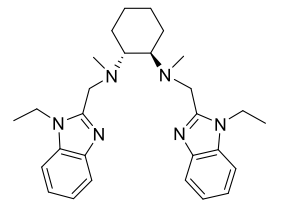

C3

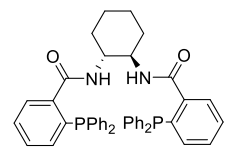

c9

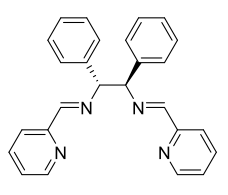

C4

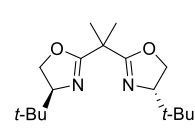

C10

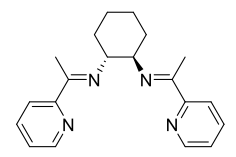

C5

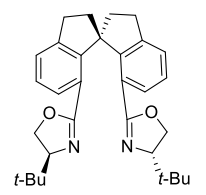

c11

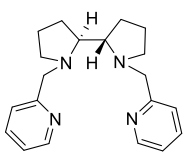

c6

Table S8. Effect of ligand structure

\begin{tabular}{|l|l|c|c|c|}
\hline entry & ligand & assay yield $^{a}$ & conversion $^{a}$ & d.r. $^{a}$ \\
\hline 1 & C1 & $78 \%$ & $91 \%$ & $88: 12$ \\
\hline 2 & C2 & $81 \%$ & $100 \%$ & $90: 10$ \\
\hline 3 & C3 & $2 \%$ & $9 \%$ & $83: 17$ \\
\hline 4 & C4 & $<1 \%$ & $6 \%$ & --- \\
\hline 5 & C5 & $<1 \%$ & $3 \%$ & --- \\
\hline 6 & C6 & $40 \%$ & $48 \%$ & $89: 11$ \\
\hline 7 & C7 & $<1 \%$ & $3 \%$ & --- \\
\hline 8 & C8 & $<1 \%$ & $4 \%$ & --- \\
\hline 9 & C9 & $<1 \%$ & $7 \%$ & --- \\
\hline 10 & C10 & $<1 \%$ & $13 \%$ & --- \\
\hline 11 & C11 & $<1 \%$ & $10 \%$ & --- \\
\hline 12 & C12 & $<1 \%$ & $11 \%$ & --- \\
\hline
\end{tabular}

assayed after $\mathrm{H}_{2} \mathrm{O}_{2}$ slow addition complete (1 h)

${ }^{1}$ Beaver, M.; Cui, S.; Xianqing, S. WO 2018/027021A1. 